\section{Low birth weight - a predictor for psychiatric problems?}

Sir - Pasamanick and colleagues first discovered a relationship between intrauterine complications and the possibility of psychiatric sequelae during the $1950 \mathrm{s.}$ Subsequently, Pasamanick proposed a 'continuum of reproductive casualty' purporting possible outcomes to an infant from a traumatic delivery could range from reading disorders and abnormal behaviour, to profound disability and death. ${ }^{2}$

Following these observations, subsequent research began to focus on the association between perinatal risk and the development of psychiatric disturbances in children. However, investigations in this area were fraught with uncertainty due to the ambiguous definition of perinatal risk. This appellation is dependent upon the gestational age of the foetus, which in its self, is an inaccurate measurement. Consequently, over the past decade research has focused upon the correlation between birth weight and abnormal childhood development. There are distinct advantages to this method of investigation; birth weight is an extremely simple yet accurate measurement, and low birth weight has been established to correlate well with perinatal risk factors, such as intrapartum complications and perinatal disease.

Sommerfelt et al examined the behavioural differences in five year old children from two cohorts. ${ }^{3}$ The first group contained 217 children of birth weights exceeding $3 \mathrm{~kg}$, and the second group contained 217 children who weighed less than $2 \mathrm{~kg}$ at birth. Questionnaires were distributed to the mothers of the children so as to obtain their perspective of their child's behaviour. There was also a three hour behavioural examination of the child undertaken by independent examiners, and a consultant paediatrician performed an attention deficit and hyperactivity assessment of the child. The results of this study revealed $19 \%$ of the below $2 \mathrm{~kg}$ birth weight children (study group) had behavioural problems compared with $4 \%$ of the control group. These behavioural difficulties relate to being more anxious, difficult to handle, impulsiveness and having a lower attention span.

Following on from this research Sykes and co-workers compared a cohort of 243 seven to eight year olds, who had been born prematurely, having a very low birth weight $(<1.5 \mathrm{~kg})$ to normal birth weight $\left(>2.5 \mathrm{~kg}\right.$ ) controls. ${ }^{4}$ They interviewed the mothers and school teachers of these children in order to assess any behavioural disparity. Regarding the mother's perception of her own child's behaviour, no significant difference was found between the control and study groups. However, the teachers' reports indicated that the very low birth weight children (in both sexes) showed more aggressive behaviour, were not as attentive, were socially less well adapted, and complained of more somatic ailments. This study was controlled for socioeconomic status and maternal age between the two cohorts.

While both these studies agree that behavioural differences exist between low birth weight and normal birth weight children, the authors refrain from using any psychiatric disorder label. Sykes and colleagues argue that the attention deficits, social withdrawal and hyperactivity symptoms are only mild to medium in nature, and do not fit any diagnostic criteria. ${ }^{4}$ However, Botting et al clearly state that low birth weight is associated with attention deficit-hyperactivity disorder (according to DSM classification). ${ }^{5}$ The investigation conducted by Botting was in children of 12 years of age, therefore older than those in the trials by Sommerfelt and Sykes. ${ }^{3 ., 5}$ This has led to speculation that as the age of the child increases these symptoms become progressively more severe, and hence, a specific diagnosis may then be applied.

The advances in neonatal medicine over the past two decades have ensured the survival of low birth weight children. The preponderance of antecedent literature focuses on behavioural abnormalities in these children. However, it is possible that while the number of low birth weight children increase there will be an associated increase in psychiatric disorders. This will lead to implications not only for medicine but for society as a whole.

I carried out a study is to investigate whether low birth weight is associated with psychiatric disorders in children and adolescents. Examination of patient records at a child and adolescent psychiatric unit in Leeds revealed birth details from which we can establish whether children and adolescents of low birth weight $(<2.5 \mathrm{~kg})$ have a higher probability of being admitted to a psychiatric unit compared to normal birth weight children.

Letters were sent to the parents of 12 children and six adolescents from a psychiatric unit in Leeds. This letter asked for the permission for their child's birth details to be used in this study, and the actual birth weight of their child. The age, sex, birth weight and reason for referral to a psychiatric unit, were recorded for all the participants. Birth weight in pounds and ounces was converted into grams by the following standard conversions; ounces to grams, multiply by 38.35 ; pounds to grams, multiply by 453.6. Data were analysed using confidence intervals following a Poisson distribution to a $95 \%$ confidence level. This was done via the computer package Arcus Qu.stat.2000.

The results are based on 18 subjects. However, one patient was attending medical inpatients at the time of data collection and was therefore omitted from the trial. Five parents did not return the questionnaire and therefore five further participants were excluded from the results. This left a total of 12 subjects.

Three of the 12 subjects were classified in the low birth weight category. This gave a prevalence of $25 \%$. Using the statistical package previously described, the $95 \%$ confidence interval for this value is between $5.4 \%$ and $57.2 \%$. The median year for date of birth for the trial participants falls between 1990 and 1991. An accepted value for the incidence of low birth weight at this time in the general population is $7.1 \% .^{6}$

The results indicate that the incidence of low birth weight in the general population fits into the $95 \%$ confidence interval for the sample population. This means that we cannot reject the null hypothesis and therefore conclude that low birth weight is a predictor for child and adolescent psychiatric problems. However, it is possible to obtain a corollary from this data. Even though the sample size in this study was small the statistical method of analysis took this into account, and yet the general incidence of low birth weight still fell on the very border of the confi- 
dence interval $(7.1 \%$ compared to $5.4 \%)$.

Explanations for psychiatric sequelae in the low birth weight infant are somewhat nebulous. The majority of low birth weight children are born prematurely, ie. are born before 37 weeks of gestation. Therefore, many of these infants have immature nervous systems. Following on from the postulation by Field,' that birth is an extremely stressful event for the newborn, Duffy et al, ${ }^{8}$ surmise that the low birth weight infants unlike their normal counterparts have development of the nervous system in a stressful extra-uterine environment. Duffy and co-workers ratiocinate that their work and that of Field's is the explanation for behavioural disturbances in these low birth weight infants.

Eckerman and colleagues, ${ }^{9}$ extrapolated the findings of Duffy et al. They propose that due to the comportment abnormalities in these children, the parenting is sub-optimal. This is because they smile less and show little interest in the mother; therefore the mother has less interaction with the child, thereby exacerbating the conduct anomalies. This can ultimately extend to the behaviour observations in low birth weight children by Sommerfelt et $a l,{ }^{3}$ and Sykes $e t a l,{ }^{4}$ and to the possibility that, as these infants mature, these insidious problems may develop into psychiatric complications described by Botting. ${ }^{s}$

However, a more organic cause for conduct disturbances in these children is the increased risk of perinatal injury of low birth weight infants in utero. Following the introduction of ultrasonography in the early 1980 s it was possible to non-invasively observe the brain of the unborn child. However, the preponderance of research focused on motor and cognitive sequelae. Using this technique Whitaker $e t$ al studied 1,105 infants with a birth weight below $2,000 \mathrm{~g} \cdot{ }^{10} \mathrm{Then}$, they followed up these children at six years of age to establish any behavioural or psychiatric abnormality. The conclusions drawn from this prospective cohort study reveal that those who had an abnormal cranial ultrasound had a four fold risk of developing a psychiatric disorder.

The specific neuroanatomical abnormality which correlated with psychiatric sequelae was injury to the perinatal white matter. ${ }^{10}$ This white matter is situated below the cortex and contains the myelinated axons of nerve processes. ${ }^{11}$ These nerve processes can be classified into three groups; association fibres which interconnect cortical sites within one cerebral hemisphere; commissural fibres which connect functionally related structures between the two hemispheres; and projection fibres which pass between the cerebral cortex and subcortical structures such as the thalamus, striatum, brain stem and spinal cord. In the neonate the subcortical white matter has a single arterial supply and is therefore extremely sensitive to ischaemic injury. The arterial damage causes bleeding into, and thus enlargement of, local ventricles. Whitaker and colleagues discovered that infants with parenchymal lesions and ventricular enlargement, processes indicative of white matter ischaemic insult, are those who go onto suffer psychiatric sequelae. ${ }^{10}$ Following 32 weeks gestation the arterial supply of this region increases and hence it is at less risk from hypoperfusion. Therefore, it is only premature infants who usually suffer these complications with subcortical white matter injury. These are also the cohort characterised by low birth weight.

However, white matter lesions are correlated with abnormalities of the basal ganglia, brain stem and cere- bellum. ${ }^{10}$ It is also argued that cortical grey matter may also be affected by white matter injury due to late migration, organisation, and myelination in the development process. ${ }^{10}$ Hence, it may not be affected white matter which causes psychiatric disturbances in the child and adolescent, but rather other regions which have been affected by white matter lesions after cranial ultrasonography was performed.

The study is not without limitation. The small sample population makes interpretation, even in the face of valid statistical analysis, conjectural. Many confounding variables have not been taken into account in this study. For example, an infant born to parents of low socio-economic class and racial minority has an increased likelihood of being low in birth weight. ${ }^{2}$ This is because these two groups are associated with decreased maternal age; increased parity; a reduction in birth interval; higher rates of infection; greater tobacco and alcohol consumption in general and during pregnancy; and poor nutrition. Coupled with this is the greater tendency for these two coterie to have negative influences on cognitive and emotional function. ${ }^{12}$

To ascertain birth weight the only form of data collection was the ability of the parents to remember this value in pounds and ounces. Obstetric records of the weight in grams were not obtained. This may be regarded as a possible source of error, although it is well recognised that your child's birth weight is a fact you rarely forget like your own date of birth. Then the multiplication to give the figure in grams could theoretically lead to loss of accuracy. However, this loss of accuracy would be extremely marginal. Nevertheless, a future recommendation for possible advancement on this research has to be the gathering of obstetric records.

While it would be injudicious to conclude that low birth weight is associated with psychiatric disorders in children or adolescents from this exploratory study, it would be equally imprudent to disregard it. This novel investigation should be used as a basis for other research to follow. In order to answer the question - 'Is low birth weight a predictor for child and adolescent psychiatric problems?' - these results need to be replicated and examined with greater methodological rigor, especially with respect to the accuracy of birth weight measurement.

Any future research in this area would have to prove sufficient statistical power to establish the presence or absence of an association with any level of statistical certainty. Further research would ideally be in the form of a prospective birth cohort, with prospective case ascertainment, or as a well powered case control study with the ability to match and control for important confounding variables. ${ }^{13}$ Given that the rates of low birth weight will continue to increase with the advances in medical technology, early interventions in high risk children to improve behaviour and reduce psychiatric sequelae would be extremely beneficial.

\section{Andrew Harris, BSc (Hons.) Fourth year medical student University of Leeds.}

References

1. Laucht M, Esser G, Baving L, Gerhold M, Hoesch I. Behavioural sequelae of perinatal insults and early family adversity at 8 years of age. J Am Acad Child Adolesc Psychiatry 2000; 39(10): 1229-37.

2. Breslau N. Psychiatric sequelae of low birth weight. Epidemiol Rev 1995; $17(1): 96-106$.

3. Sommerfelt K, Troland K, Ellertsen B, Markestad T. Behavioural problems 
in low-birth weight preschoolers. Dev Med Child Neurology 1996; 38: 927-40. 4. Sykes DH, Hoy EA, Bill JM, McClure BG. Halliday HL. Behavioural adjustment in school of very low birth weight children. J Child Psychol Psychiat $1997 ; 38(3): 315-25$

5. Botting N, Powls A, Cooke RW. Attention deficit hyperactivity disorders and other psychiatric outcomes in very low birth weight children at 12 years. J. Child Psychol Pyschiat 1997; 38(8): 931-41.

6. Behrman RE, Kliegman RM, Aruin AM. In: Nelson textbook of Pediatrics, 15th Edition. W.B. Saunders Company London, 1996; 78: 431-33.

7. Field TM. Neonatal stress and coping in intensive care. Infant Mental Health J 1990; 11:57-65.

8. Duffy FH, Als H, McAnulty GB. Behavioural and electrophysiological evidence for gestational age effects in healthy preterm and full-term infants during the first year of life. Child Development 1990; 61: 1271-86.
9. Eckerman CO, Oehler JM, Medvin MB, Hannan TE. Premature newborns as social partners before term age. Infant Behaviour and Development 1994; $17: 55-70$.

10. Whitaker AH, Rossem RV, Feldman JF, Schonfeld IS, Pinto-Martin JA. Psychiatric outcomes in Low-birth-weight children at 6 years: relation to neonatal cranial ultrasound abnormalities. Arch Gen Psychiatry 1997; 54: 847 56.

11. Crossman AR, Neary D. In: Neuroanatomy an illustrated colour text Churchill Livingstone Edinburgh, 1995; 114-7.

12. Breslau N, Brown GG, DelDotto JE, Kumar S, Ezhuthachan S. Psychiatric sequelae of low birth weight at 6 years of age. J. Abnorm. Child Psychol 1996; 24(3): $385-400$

13. Hennekens $\mathrm{CH}$, Buring JE. In: Epidemiology in Medicine. Little, Brown and Company, Boston, 1987

\section{BOOK REVIEWS}

Ir J Psych Med 2001; 18(3): 112

\section{Bipolar medications: mechanisms of action}

Edited by HK Manji, CL Bowden, RH Belmaker. American Psychiatric Press Inc., Washington DC., 2000. Pgs440. ISBN 0-88048-927-8.

Lithium was one of the first drugs to herald the psychopharmacological revolution some 50 years ago and it still remains one of the standard treatments for bipolar disorder. The complexity of action of lithium at the cellular level still remains an enigma despite all the advances in molecular neuroscience over the past decade. Undoubtedly the inability to patent lithium, combined with its success in the treatment of patients with bipolar disorder, contributed to the relative lack of interest of the pharmaceutical companies in the development of drugs to treat bipolar disorder. Thus neuroleptics to treat the acute symptoms of the disturbed patient, together with lithium for the long term maintenance, largely formed the basis of the drug therapy for bipolar disorder for the past 40 years. Meanwhile, other areas of psychopharmacology developed at an astounding rate with the introduction of novel antidepressants, antipsychotics and anxiolytics.

Despite the relative neglect of bipolar disorder by the pharmaceutical industry in the past, there has recently been a revival of interest for two main reasons. First because bipolar disorder is one of the few psychiatric conditions in which there is a genetic basis . In addition, its cyclical nature gave researchers the opportunity to study the transition from mania to depression, a situation which was not nearly so well defined in any other condition. Second, a number of psychotropic drugs were developed which were found to be therapeutically effective in the treatment of the disorder and which were superior to lithium in terms of their tolerability, safety and acceptability to the patient. The present volume provides an excellent summary of the types of drug now available and of their proposed mechanism of action.

Bipolar Medications consists of 19 chapters whose contributors are among the leading researchers in bipolar disorder. Eight of the chapters are concerned with different aspects of the pharmacology and biochemistry of lithium. The pharmacological properties of carbamazepine and valproate are considered in two chapters while lamotrigine and calcium channel blockers are each discussed in separate chapters. In brief, this volume emphasises future progress which may arise from the basic clinical and experimental studies now being undertaken. For example, Chapter 1 recounts the evidence to suggest that lithium works via the inhibition of inositol monophosphatase and this provides the impetus for developing drugs which specifically inhibit this enzyme. So far, however, the substrate based inhibitors have been found to have only limited activity. The effect of lithium on guanine nucleotide binding proteins (G-proteins) is also considered and while the action of drugs apart from lithium on G-proteins has not resulted in new drugs, it is evident that many second messenger systems such as protein kinase $\mathrm{C}$, are affected by lithium, valproate and carbamazepine. It has been suggested for example, that the short-term effects of lithium are due to its modulatory effects on second signalling systems and the consequent expression of specific genes. Long-term benefits are however derived from the down-regulation of pro-apoptotic signalling molecules and changes in the cytoskeleton. With regard to the pharmacological actions of lithium, valproate and carbamazepine, Post and colleagues have provided a particularly valuable review of the current situation.

The clinical chapters provide a valuable update of the problems in the field. Bowden, for example, illustrates the importance of valproate as an alternative to lithium while Calabrese reviews the evidence that lamotrigine may be particularly useful in the treatment of the depressive phase of bipolar disorder. The possibility of replacing typical neuroleptics by atypical agents such as olanzapine is considered by Tohen and Tollefson while Goodwin raises the problems which can arise following the abrupt discontinuation of lithium after a relatively short (two years) period of treatment. In conclusion, this monograph provides an excellent overview of the pharmacology and clinical applications of the standard drugs used to treat bipolar disorder and as such should be an essential addition to all departmental libraries. It is a pity that some of the more recently discovered drugs such as gabapentin and topiramate are not mentioned, as these drugs, by their different mechanisms of action, may help to throw light on the biological complexity of bipolar disorder and thereby assist in the development of new concepts for drug treatment.

Brian E Leonard, Pharmacology Department, National University of Ireland, Galway, Ireland. 